\title{
Transverse coherence and pointing stability of the radiation from x-ray free electron lasers
}

\author{
E. A. Schneidmiller and M.V. Yurkov \\ Deutsches Elektronen-Synchrotron (DESY), Notkestr. 85, D-22607 Hamburg, Germany
}

\begin{abstract}
The radiation from SASE FEL has always limited value of the degree of transverse coherence. When transverse size of the electron beam significantly exceeds diffraction limit, the mode competition effect does not provide the selection of the ground mode, and spatial coherence degrades due to contribution of the higher order transverse modes. It is important that the most strong higher modes are azimythally non-symmetric which leads to fluctuations of the spot size and of the pointing stability of the photon beam. These fluctuations are fundamental and originate from the shot noise in the electron beam. The effect of the pointing instability becomes more pronouncing for shorter wavelengths. We analyze in detail the case of optimized SASE FEL and derive universal dependencies applicable to all operating and planned x-ray FELs. It is shown that x-ray FELs driven by low energy electron beams will exhibit poor spatial coherence and bad pointing stability.
\end{abstract}

Keywords: X-ray, Free Electron Laser, Undulator, Coherence

\section{INTRODUCTION}

Coherence properties of the radiation from SASE FEL strongly evolve during the amplification process. ${ }^{2-6}$ At the initial stage of amplification the spatial coherence is poor, and the radiation consists of a large number of transverse modes. ${ }^{6-14}$ In the exponential stage of amplification the transverse modes with higher gain dominate over modes with lower gain when the undulator length progresses. The mode selection process stops at the onset of the nonlinear regime, and the maximum values of the degree of the transverse coherence is reached at this point.

Gain separation of the FEL radiation modes is mainly defined by the value of the diffraction parameter $B^{11}$ describing diffraction expansion of the radiation with respect to the electron beam size. For FEL with diffraction limited beams the value of the diffraction parameter is less or about unity, and spatial coherence at saturation reaches values about $90 \%$. Large values of the diffraction parameter $(B \gtrsim 10 \ldots 100)$ are typical for SASE FELs operating in the hard $\mathrm{x}$-ray range. ${ }^{15-19}$ Increase of the diffraction parameter results in a decrease of diffraction expansion and of relative separation of the gain of the modes. In this case we deal with the mode degeneration effect. ${ }^{8,11}$ Since the number of gain lengths to saturation is limited (about 10 for x-ray FELs), the contribution of the higher spatial modes to the total power grows with the value of the diffraction parameter, and the transverse coherence degrades.

The main competitor of the ground $\mathrm{TEM}_{00}$ mode is the first azimuthal $\mathrm{TEM}_{10}$ mode. When contribution of $\mathrm{TEM}_{10}$ mode to the total power exceeds a few per cent level, a fundamental effect of bad pointing stability becomes to be pronouncing. For optimized SASE FEL the power of the effect grows with the parameter $\hat{\epsilon}=$ $2 \pi \epsilon / \lambda$. SASE FELs operating at short wavelengths and low electron beam energy with the value of $\hat{\epsilon} \gtrsim 1$ suffer from the mode degeneration effect resulting in significant degradation of the spatial coherence and pointing stability of the photon beam. The effect of the photon beam pointing jitter is a fundamental one, and can not be eliminated by eliminating of the jitters of machine parameters (orbit, phase, etc.). 


\section{MODE DEGENERATION EFFECT}

The amplification process in SASE FEL starts from the shot noise in the electron beam. In the exponential stage of amplification the radiation consists of a large number of transverse and longitudinal modes: ${ }^{6-14}$

$$
\tilde{E}=\sum_{m, n} \int \mathrm{d} \omega A_{m n}(\omega, z) \Phi_{m n}(r, \omega) \exp \left[\Lambda_{m n}(\omega) z+i m \phi+i \omega(z / c-t)\right]
$$

Each mode is characterized by the eigenvalue $\Lambda_{m n}(\omega)$ and the field distribution eigenfunction $\Phi_{m n}(r, \omega) .{ }^{9,10}$ The

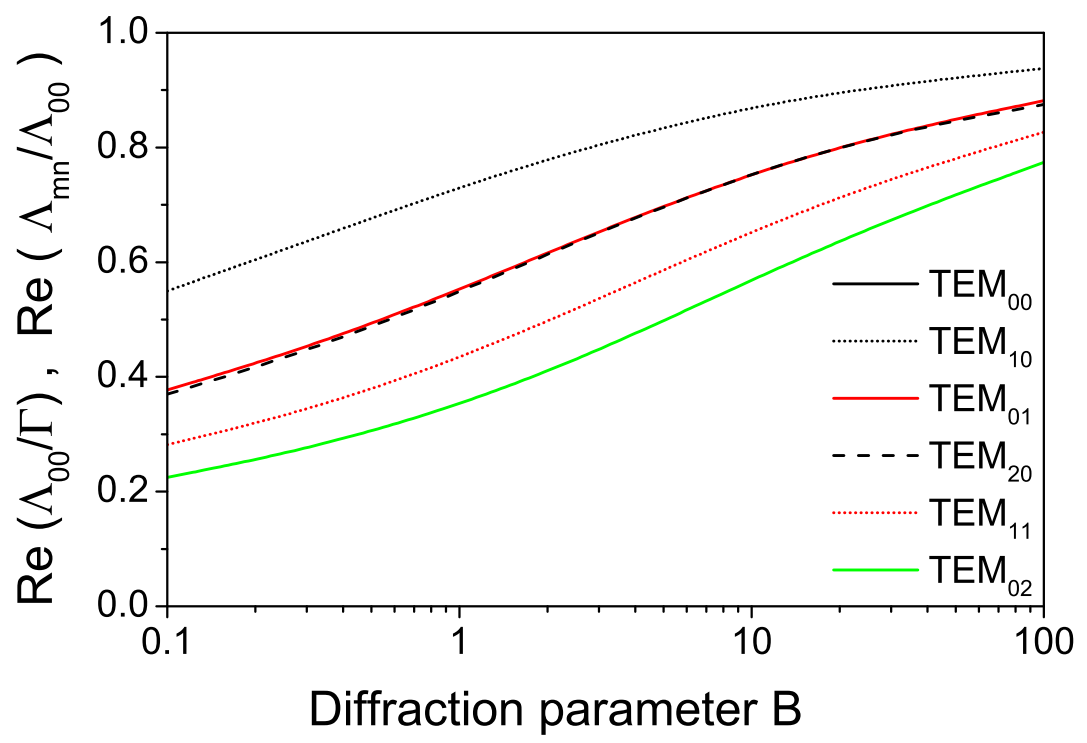

Figure 1. The ratio of the maximum gain of the higher modes to the maximum gain of the fundamental mode $\operatorname{Re}\left(\Lambda_{m n}\right) / \operatorname{Re}\left(\Lambda_{00}\right)$ versus diffraction parameter $B$ for the case of "cold" electron beam. Calculations have been performed with code FAST. ${ }^{23}$

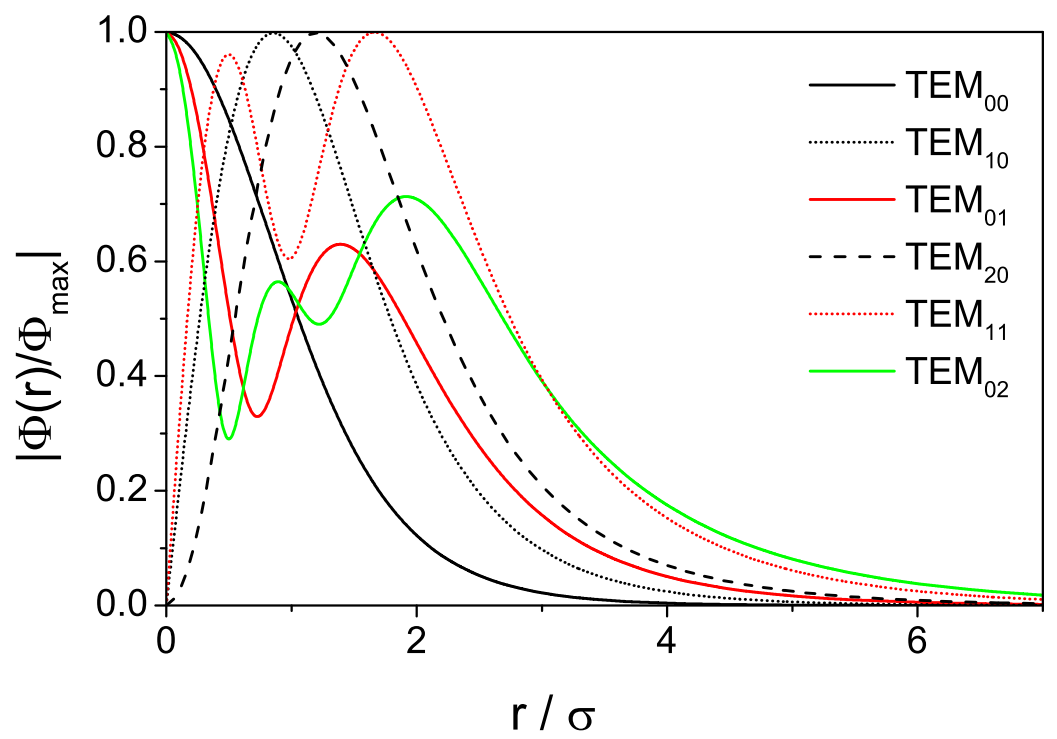

Figure 2. Aamplitude of the eigenfunctions of the FEL radiation modes, $\left|\Phi_{m n}(r)\right| /\left|\Phi_{\max }\right|$ for diffraction parameter $B=10$. The list of modes is ranked by the gain. Calculations have been performed with code FAST. ${ }^{23}$ 
real part of the eigenvalue $\operatorname{Re}\left(\Lambda_{m n}(\omega)\right)$ is referred to as the field gain. Fundamental $\mathrm{TEM}_{00}$ mode has the highest gain. Relative gain of its nearest competitors is depicted in Fig. 1 as function of the diffraction parameter. Sorting the modes by the gain results in the following ranking: $\mathrm{TEM}_{00}, \mathrm{TEM}_{10}, \mathrm{TEM}_{01}, \mathrm{TEM}_{20}, \mathrm{TEM}_{11}, \mathrm{TEM}_{02}$. The difference in the gain between the fundamental $\mathrm{TEM}_{00}$ mode and higher modes is pronounced for small values of the diffraction parameter $B \lesssim 1$, but it falls down for large values of $B$. In other words, the effect of the mode degeneration takes place. Its origin can be understood with the qualitative analysis of the eigenfunctions (see Figure 2). For small values of the diffraction parameter the field of the higher spatial modes spans far away from the core of the electron beam while the fundamental $\mathrm{TEM}_{00}$ mode is more confined. This feature provides a higher coupling factor of the radiation with the electron beam and higher gain. For large values of the diffraction parameter all radiation modes shrink to the beam axis which results in an equalizing of coupling factors and of the gain. Asymptotically, the eigenvalues of all modes tends to the one dimensional asymptote as: ${ }^{4}$

$$
\Lambda_{m n} / \Gamma \simeq \frac{\sqrt{3}+\mathrm{i}}{2 B^{1 / 3}}-\frac{(1+\mathrm{i} \sqrt{3})(1+n+2 m)}{3 \sqrt{2} B^{2 / 3}}
$$

where $\Gamma$ is the gain parameter. ${ }^{11}$ For x-ray SASE FEL, the undulator length to saturation is about 10 field gain lengths. ${ }^{2,3,5}$ The mode selection process stops at the onset of the nonlinear regime, about two field gain lengths before saturation. For the value of the diffraction parameter $B=1$ we get from Fig. $1 \operatorname{Re}\left(\Lambda_{10} / \Lambda_{00}\right)=0.73$, and the ratio of field amplitudes of $\mathrm{TEM}_{00}$ and $\mathrm{TEM}_{10}$ modes exceeds a factor of 10 at the onset of the nonlinear regime. For $B=10$ we have $\operatorname{Re}\left(\Lambda_{10} / \Lambda_{00}\right) \simeq 0.87$, and the ratio of the field amplitudes is about of factor of 3 only. An estimate for the contribution of the higher spatial modes to the total power is about $10 \%$. Thus, an excellent transverse coherence of the radiation is not expected for SASE FEL with diffraction parameter $B \gtrsim 10$.

\section{OPTIMIZED SASE FEL}

Target value for x-ray FEL optimization is the gain of the fundamental mode. In this case the field gain length of the fundamental mode and optimum beta function, are rather accurately approximated by: ${ }^{2,3,21}$

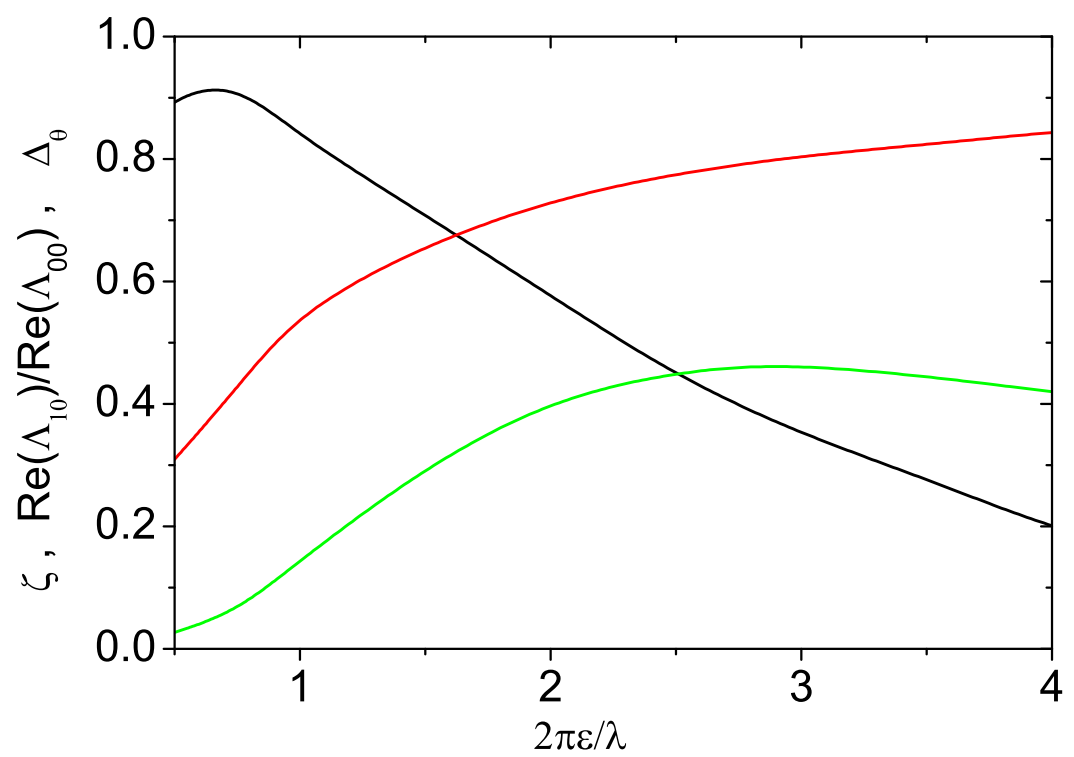

Figure 3. Optimized SASE FEL: Degree of transverse coherence $\zeta$ (black curve), ratio of the gain $\operatorname{Re}\left(\Lambda_{10}\right) / \operatorname{Re}\left(\Lambda_{00}\right)(\operatorname{red}$ curve), and rms deviation of the photon beam center of gravity $\Delta_{\theta}$ in terms of rms size of the photon beam (green curve). Simulations have been performed with code FAST. ${ }^{23}$ 


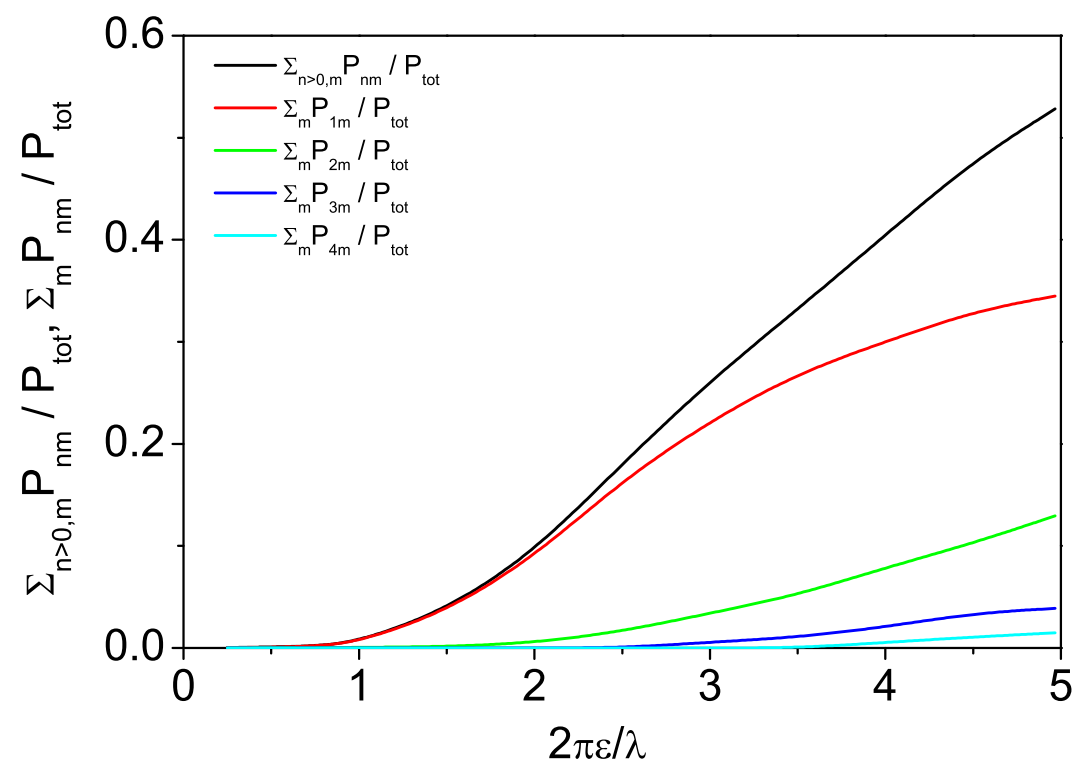

Figure 4. Optimized SASE FEL: partial contributions of nonsymmetric modes to the total power versus the emittance parameter $\hat{\epsilon}=2 \pi \epsilon / \lambda$. SASE FEL operates in the saturation. Black curve is total contribution of nonsymmetric modes, and color curves correspond to azimuthal indices from 1 to 4 . Simulations have been performed with code FAST. ${ }^{23}$
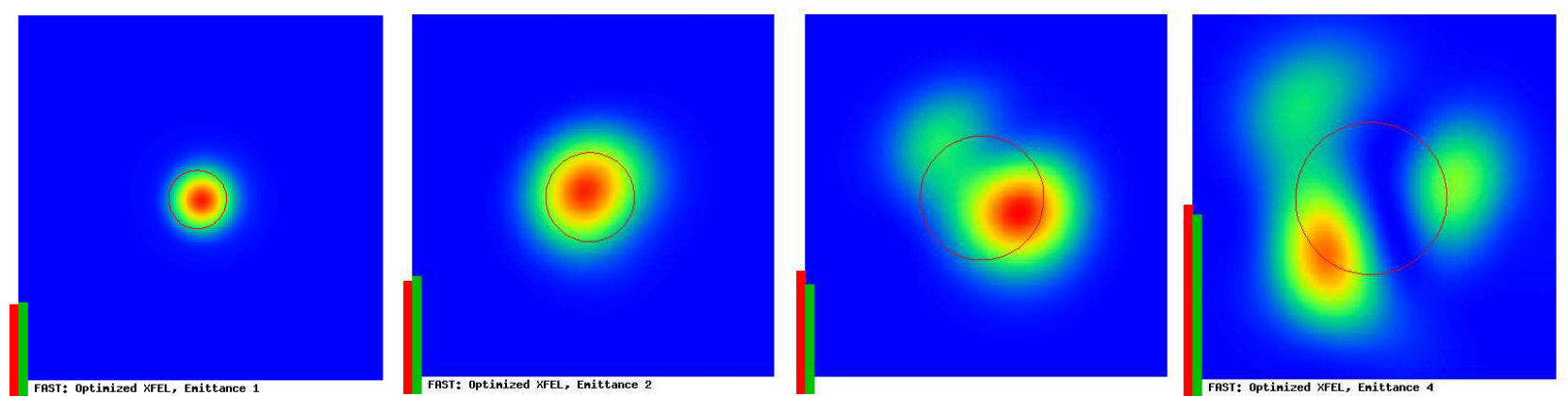

Figure 5. Typical slice distribution of the radiation intensity for optimized SASE FEL with $\hat{\epsilon}=1,2,3,4$ (from left to right). Circle denotes rms spot size. SASE FEL operates in the saturation. Simulations have been performed with code FAST. $^{23}$

$$
\begin{aligned}
L_{\mathrm{g}} & =1.67\left(\frac{I_{A}}{I}\right)^{1 / 2} \frac{\left(\epsilon_{n} \lambda_{\mathrm{w}}\right)^{5 / 6}}{\lambda^{2 / 3}} \frac{\left(1+K^{2}\right)^{1 / 3}}{K A_{J J}}(1+\delta) \\
\beta_{\mathrm{opt}} & \simeq 11.2\left(\frac{I_{A}}{I}\right)^{1 / 2} \frac{\epsilon_{n}^{3 / 2} \lambda_{\mathrm{w}}^{1 / 2}}{\lambda K A_{J J}}(1+8 \delta)^{-1 / 3} \\
\delta & =131 \frac{I_{A}}{I} \frac{\epsilon_{n}^{5 / 4}}{\lambda^{1 / 8} \lambda_{\mathrm{w}}^{9 / 8}} \frac{\sigma_{\gamma}^{2}}{\left(K A_{J J}\right)^{2}\left(1+K^{2}\right)^{1 / 8}}
\end{aligned}
$$

where $\sigma_{\gamma}=\sigma_{\mathrm{E}} / m c^{2}, \sigma_{\mathrm{E}}$ is rms energy spread, $\epsilon_{n}=\gamma \epsilon$ is normalized rms emittance, $K$ is rms undulator parameter, $A_{J J}$ is averaging factor, $\lambda$ is radiation wavelength, $\lambda_{\mathrm{w}}$ is undulator period, $I$ is the beam current, $I_{A} \simeq 17 \mathrm{kA}$ is Alfven's current.

In the case of negligibly small energy spread, characteristics of SASE FEL written down in the dimensionless form are functions of two parameters, $\hat{\epsilon}=2 \pi \epsilon / \lambda$ and parameter $N_{\mathrm{c}}=I L_{\mathrm{g}} \lambda /\left(e \lambda_{\mathrm{w}} c\right)$ defining the initial conditions for the start-up from the shot noise. ${ }^{2-4}$ Dependence of characteristics on the value of $N_{\mathrm{c}}$ is slow, in fact 
Table 1. Parameter space of x-ray FELs

LCLS SACLA EXFEL SWISS FEL PAL XFEL

$\begin{array}{lccccc}\text { Energy }[\mathrm{GeV}] & 13.6 & 8.0 & 17.5 & 5.8 & 10 \\ \text { Wavelength }[\mathrm{A}] & 1.5 & 0.6 & 0.5 & 0.7 & 0.6 \\ \epsilon_{n}[\mathrm{~mm}-\mathrm{rad}] & 0.4 & 0.4 & 0.4 & 0.4 & 0.4 \\ \hat{\epsilon} & 1 & 2.7 & 1.5 & 3.4 & 2.1\end{array}$

logarithmic. The diffraction parameter scales with the emittance parameter as $B \simeq 13 \times \hat{\epsilon}^{5 / 2}$. Starting from $\hat{\epsilon}>1$ the gain of the TEM 10 mode approaches very close to the gain of the ground $\mathrm{TEM}_{00}$ mode (see Fig. 3 ). Contribution of the $\mathrm{TEM}_{10}$ mode to the total power progresses with the growth of the emittance parameter (see Fig. 4). Starting from $\hat{\epsilon}>2$ the azimuthal modes $\mathrm{TEM}_{2 n}$ appear in the mode contents, and so on. The maximum value of the degree of transverse (which occurs in the end of the linear regime) degrades gradually with the growth of the emittance parameter (see Fig: 3).

Mode degeneration has significant impact on the pointing stability of SASE FEL. Figure 4 shows the relative contribution to the total radiation energy of the modes with higher azimuthal indices. Typical intensity distributions in the far zone are shown in Fig. 5. Transverse intensity patterns in slices have a rather complicated shape due to the interference of the fields of statistically independent modes with different azimuthal indices. These slice distributions are essentially non-gaussian when relative contribution of higher azimuthal modes to the total power approaches $10 \%$. The shape of the intensity and phase distributions change drastically on a scale of the coherence length, and source point position and pointing jumps from spike to spike. Figure 3 represents quantitative description of this phenomena using notion of the rms deviation of the photon beam center of gravity $\Delta_{\theta}$ expressed in terms of the rms size of the photon beam. We see that there is no perfect pointing of the photon beam, and for the values of $\hat{\epsilon} \gtrsim 2$ fluctuations of the pointing exceed $40 \%$. Averaging of slice distributions over a radiation pulse results in a more smooth distribution. However, with limited number of longitudinal modes, the center of gravity of the radiation pulse (position) and its shape jitter from shot to shot which is frequently referred to as poor pointing stability. This effect is experimentally observed at FLASH, ${ }^{22}$ and evidently it should take place at other x-ray facilities. Only in the case of a long radiation pulse, or after averaging over many pulses, the intensity distribution approaches asymptotically to an azimuthally symmetric shape.

Table 1 presents list of parameters of the x-ray FELs compiled for the shortest design wavelength. ${ }^{15-19}$ We assume the normalized emittance to be the same for all cases $\left(\epsilon_{n}=0.4 \mathrm{~mm}-\mathrm{mrad}\right)$. Lower energy of electron beam results in larger value of the emittance parameter, and output radiation will have poor spatial coherence and poor pointing stability of the photon beam. Note that spatial jitter is of a fundamental nature (shot noise in the electron beam), and takes place even for an 'ideal' machine.

There are only very limited means to suppress the mode degeneration effect by means of controlling the spread of longitudinal velocities (due to energy spread and emittance). ${ }^{1,8,11}$ Energy spread can be increased with the laser heater. ${ }^{24}$ The price for this improvement is significant increase of the saturation length and reduction of the FEL power. Stronger focusing of the electron beam in the undulator helps to improve transverse coherence due to a reduction of the diffraction parameter and increase of the velocity spread. However, this will also result in the increase of the saturation length. Finally, with fixed energy of the electron beam, an available undulator length will define the level of spatial coherence and spatial jitter of the photon beam.

\section{REFERENCES}

1. E.A. Schneidmiller, and M.V. Yurkov, Proc. FEL2015 Conference, Daegeon, Korea, 2015, tup021.

2. E.L. Saldin, E.A. Schneidmiller, and M.V. Yurkov, Opt. Commun. 281(2008)1179.

3. E.L. Saldin, E.A. Schneidmiller, and M.V. Yurkov, New J. Phys. 12 (2010) 035010, doi: 10.1088/13672630/12/3/035010.

4. E.L. Saldin, E.A. Schneidmiller, and M.V. Yurkov, Opt. Commun. 281(2008)4727.

5. E.A. Schneidmiller, and M.V. Yurkov, Proc. FEL 2012 Conference, mopd08.pdf.

6. E.L. Saldin, E.A. Schneidmiller, and M.V. Yurkov, Opt. Commun. 186(2000)185.

7. G. Moore, Opt. Commun. 52, 46 (1984). 
8. E.L. Saldin, E.A. Schneidmiller and M.V. Yurkov, Optics Commun 97(1993)272.

9. M. Xie, Nucl. Instrum. and Methods A 445, 59 (2000).

10. E.L. Saldin, E.A. Schneidmiller and M.V. Yurkov, Nucl. Instrum. and Methods A 475, 86 (2001).

11. E.L. Saldin, E.A. Schneidmiller, M.V. Yurkov, "The Physics of Free Electron Lasers" (Springer-Verlag, Berlin, 1999).

12. K.J. Kim, Phys. Rev. Lett. 57(1986)1871.

13. J.M. Wang and L.H. Yu, Nucl. Instrum. and Methods A250(1986)484.

14. S. Krinsky and L.H. Yu, Phys. Rev. A 35(1987)3406.

15. P. Emma et al. Nature Photonics 4, 641 (2010).

16. T. Ishikawa et al., Nature Photonics 6, 540 (2012).

17. R. Ganter (Ed.), Swiss FEL Conceptual Design Report, PSI Bericht Nr. 10-04, April 2012.

18. H.S. Kang, K.W. Kim, I.S. Ko, Proc. IPAC 2014 Conf., paper THPRO019 (2014).

19. M. Altarelli et al. (Eds.), XFEL: The European X-Ray Free-Electron Laser. Technical Design Report, Preprint DESY 2006-097, DESY, Hamburg, 2006.

20. W. Ackermann et al., Nature Photonics 1(2007)336

21. E.L. Saldin, E. A. Schneidmiller, and M.V. Yurkov, Opt. Commun. 235(2004)415.

22. E.A. Schneidmiller and M.V. Yurkov, J. Mod. Optics (2015). DOI: 10.1080/09500340.2015.1066456.

23. E.L. Saldin, E.A. Schneidmiller, and M.V. Yurkov, Nucl. Instrum. and Methods A 429(1999)233.

24. E.L. Saldin, E.A. Schneidmiller, and M.V. Yurkov, Nucl. Instrum. and Methods A 528, 355 (2004). 